\title{
HIGH-REP RATE PHOTOCATHODE INJECTOR FOR LCLS *
}

\author{
X.J. Wang ${ }^{\dagger}$, M. Babzien, I. Ben-Zvi, X.Y. Chang, S. Pjerov, and M. Woodle \\ NSLS, BNL, Upton, NY 11973, USA
}

\begin{abstract}
A preliminary design of the photocathode RF gun injection system for LCLS $120 \mathrm{~Hz}$ operation will be presented in this report. The injection system includes photocathode RF gun, emittance compensation solenoid magnet, $\mathrm{Mg}$ cathode, laser and laser beam delivery system and electron beam diagnostics. The photocathode RF gun for $120 \mathrm{~Hz}$ LCLS operation is based on the BNL gun IV with several improvements, such as better cooling and vacuum pumping capabilities, and accurate alignment. The thermal calculation of the RF gun, computer simulation of its performance, and both transverse and various longitudinal laser pulse shaping techniques will be presented.
\end{abstract}

\section{INTRODUCTION}

To develop X-ray Free electron laser (FEL) as one of the possible candidates for future fourth generation light source, a multi-institute collaboration in US is working on a SASE X-ray free electron laser project (LCLS) using 15 GeV SLAC linac [1]. One of the critical R\&D items for LCLS is to develop a reliable photocathode RF gun injection system capable of producing electron beam with emittance about $1 \mathrm{~mm}$-mrad and peak current of $100 \mathrm{~A}$. As part of LCLS collaboration, National synchrotron light source (NSLS) and Brookhaven Accelerator Test Facility (ATF) is proposing to design a photo-cathode RF gun injection system which will satisfy LCLS requirements.

The proposed photocathode RF gun injection system will base on BNL Gun IV [2]. BNL GUN VI has demonstrated the capability of $50 \mathrm{~Hz}$ operation [2], and the beam quality produced by the BNL guns lead to two SASE FELs, LEUTL [3] and VISA [4], reached saturation. Improvements will be made so the proposed photocathode RF gun injector will capable of operating at $120 \mathrm{~Hz}$ reliably. In the following sections, we will first describe the specification of the new photo-injector based on the ATF operation experience. We then present thermal analysis of the Gun IV and initial improvement in cooling capability. Beam dynamics studies were performed using PARMELA, we then describe the laser system requirements, and possible transverse and longitudinal pulse shaping techniques.

\section{HIGH-REP RATE PHOTO-INJECTOR}

The photocathode RF gun injection system consists of a 1.6 cell photocathode RF gun, a single emittance

\footnotetext{
* Supported by US DOE contract DE-AC02-98CH10886 †xwang@bnl.gov
}

compensation solenoid magnet, laser system and electron beams diagnostic cubes (fig.1). We will discuss performance requirements for those components in the rest of this section.

\section{$2.1120 \mathrm{~Hz}$ Photocathode RF gun}

The Brookhaven Accelerator Test Facility (ATF) is the only multi-user facility based on photocathode RF gun injection system, it provides more 1000 hours user beam time annually. The basic parameters of the LCLS are specified according to ATF experience with special improvement for $120 \mathrm{~Hz}$ operation. Table 1 lists the RF gun parameters. The operating temperature of the RF gun specified here is $30{ }^{\circ} \mathrm{C}$ instead of traditional $45^{\circ} \mathrm{C}$. That will reduce $10 \%$ RF power consumption without introduce any complexity in the cooling water system. For a peak field of $100 \mathrm{MV} / \mathrm{m}$, about $7 \mathrm{MW}$ RF power is required.

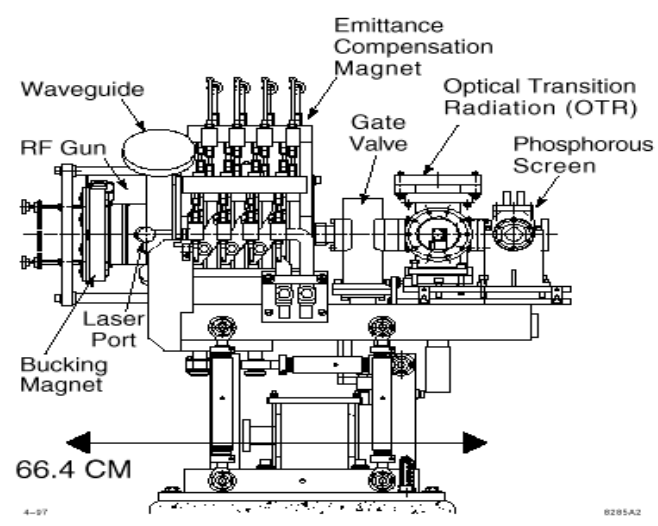

Figure 1: BNL Photo-injector system.

Table 1: $120 \mathrm{~Hz}$ RF gun

\begin{tabular}{|l|l|}
\hline $\begin{array}{l}\text { RF gun rep. Rate } \\
(\mathrm{Hz})\end{array}$ & 120 \\
\hline $\begin{array}{l}\text { Field on the cathode } \\
(\mathrm{Mv} / \mathrm{m})\end{array}$ & $100-140$ \\
\hline Cathode material & $\mathrm{Cu}$ or Mg \\
\hline $\begin{array}{l}\text { Vacuum inside the } \\
\text { gun }\end{array}$ & $\begin{array}{l}<3 \times 10^{-10} \\
\text { on }\end{array}$ \\
\hline $\begin{array}{l}\text { Operating RF } \\
\text { Temperature }\left({ }^{\circ} \mathrm{C}\right)\end{array}$ & 30 \\
\hline
\end{tabular}

Both $\mathrm{Cu}$ and $\mathrm{Mg}$ cathode were considered for our application, but our preference is $\mathrm{Mg}$ cathode. At the ATF, we have developed $\mathrm{Mg}$ cathode manufacture, preparation and cleaning technique. $\mathrm{A} \mathrm{Mg}$ cathode have been in operation at ATF for last two years at a field of 100 $\mathrm{MV} / \mathrm{m}$. For a operation vacuum better than $5 \times 10^{-10}$ Torr, 
quantum efficiency (QE) routinely reach $0.2 \%$, and $\mathrm{QE}$ variation on the cathode surface less than $10 \%(\mathrm{p}-\mathrm{p})$ after laser vacuum based cleaning.

The thermal analysis of the BNL gun IV have been extensively investigated. Figure 2 shows temperature distribution for a $3 \mathrm{KW} \mathrm{CW}$ power (corresponding to 150 $\mathrm{Hz}$ operation for a 3 us RF pulse). The hot spots are iris between the two cells and cathode. To reduce the possible thermal stress, we increase the water channel diameter by about $30 \%$, and push the water channel much closer to the hot spot. Better temperature control on the waveguide connecting to the RF gun was also added to improve the gun temperature control (Fig.3).

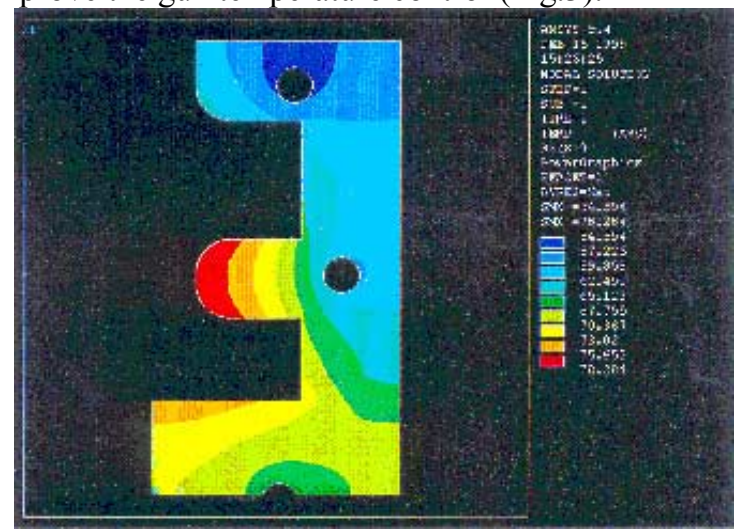

Figure 2: thermal distribution for $3 \mathrm{KW} \mathrm{CW}$ power of the Gun IV.

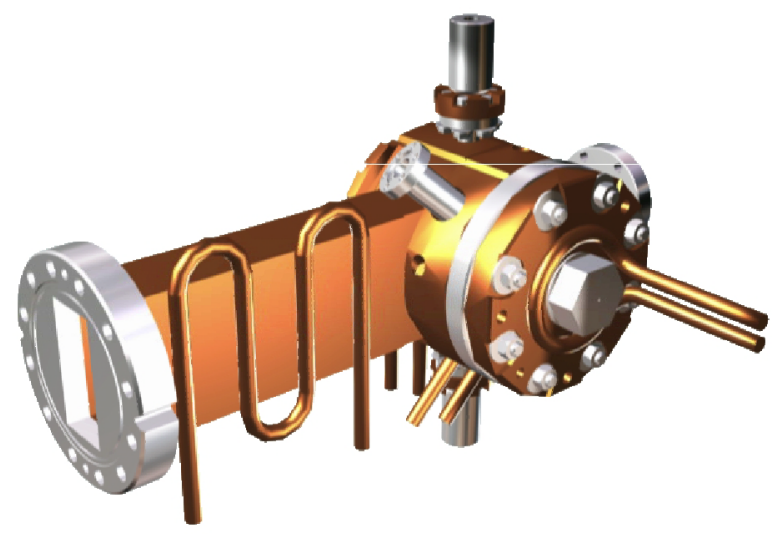

Figure 3: $120 \mathrm{~Hz}$ RF gun.

The solenoid magnet similar to Gun IV system will be used for $120 \mathrm{~Hz}$ LCLS injection system. Improvements will be made in RF gun mounting on the solenoid magnet to have better isolation and alignment. We are also considering motorize solenoid magnet support to improve the relative alignment between gun solenoid magnet assembly and boost linac to minimize the emittance growth.

The beam diagnostic cube basically consists a movable Faraday cup combine with a beam profile monitor [5]. This device is capable of determining three most basic parameters for operating a photo-injector, they are photoelectron beam charge, beam energy and relative phase between laser and RF field. A non-destructive beam charge measurement device, such as integrated current transformer (ICT) will be added for on-line beam diagnostics.

\section{$2.2120 \mathrm{~Hz}$ laser system}

Table 2 lists basic the requirements for the RF gun driving laser system. Both Ti:sapphire laser and Nd:YLF laser were considered. Our choice is Nd:YLF laser. The main reason for adopting Nd:YLF laser is the existing of $100 \mathrm{~Hz}$ diode pump laser system [6], which is inherently more stable than other technique.

The design of photo-injector can accommodate both normal and oblique laser incidents on the cathode. Normal incident has the advantage of relative simple laser optics, but in-vacuum optics made on-line laser diagnostics almost impossible, and subject to electron beam interception. Oblique laser incident will demands complex laser optics for both transverse and longitudinal wave fronts correction. On-line laser beam diagnostics will be easily implemented for qblique incident because no optics located inside the vacuum. The ATF experience shows that, oblique incident option is better choice.

Table 2: $120 \mathrm{~Hz}$ laser system

\begin{tabular}{|c|c|c|}
\hline & & \\
\hline Rep. Rate $(\mathrm{Hz})$ & 120 & \\
\hline $\begin{array}{l}\text { Laser energy on cathode } \\
(\mathrm{UV}, \mathrm{uJ})\end{array}$ & $30(\mathrm{Mg})$ & $\begin{array}{l}200 \\
(\mathrm{Cu})\end{array}$ \\
\hline $\begin{array}{l}\text { Laser pulse length (ps, } \\
\text { FWHM) }\end{array}$ & 5 to 20 & \\
\hline Laser spot (radius, mm) & $0.5-1.5$ & \\
\hline Laser energy stability (\%) & $\begin{array}{l}1.5 \\
(\mathrm{rms})\end{array}$ & $6(p-p)$ \\
\hline Timing jitter (ps) & 0.1 & $\begin{array}{l}0.5 \quad(p- \\
p)\end{array}$ \\
\hline Point stability (\%) & 0.25 & 1 \\
\hline
\end{tabular}
system are mainly determined by the laser system. The ATF laser system has demonstrated the energy stability required for the LCLS operation. The tight timing jitter requirement is mainly determined by the photo-injector operating at longitudinal emittance compensation mode which be discussed later of the paper.

One of the advantages of photocathode RF gun is space charge effect could be mitigated by proper shaping both transverse and longitudinal laser profiles. Uniformed transverse laser beam profile will be able to minimize the beam emittance. One way to realize the uniformed transverse laser beam profile is by truncation of a large Gaussian distribution. This technique is will significantly increase the laser energy requirement. A relative easy with reasonable efficiency technique is using graded mirror. Figure 4 shows preliminary experimental results of this technique being implemented at the ATF.

One of the techniques for longitudinal laser pulse shaping is using phase mask by controlling phase and amplitude modulation (Fig.5). Preliminary results are very promising [8]. Saturable absorber is another technique under consideration, it truncates the laser pulse longitudinally. It is relative simple. 

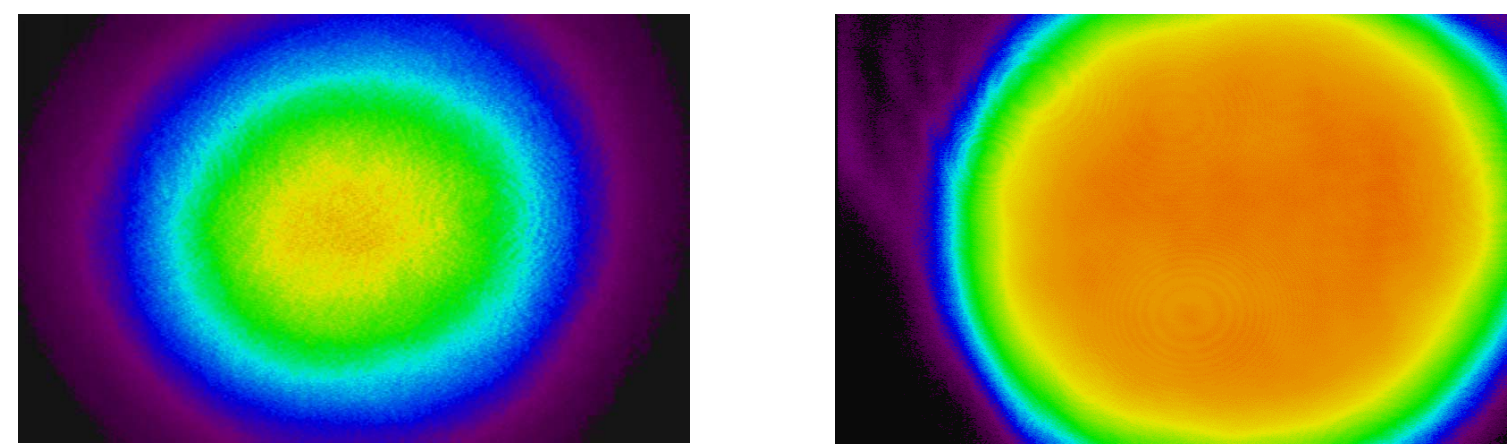

Figure 4: IR laser profile shaping using grade mirror. Left is gaussian input transverse profile, right is the output.

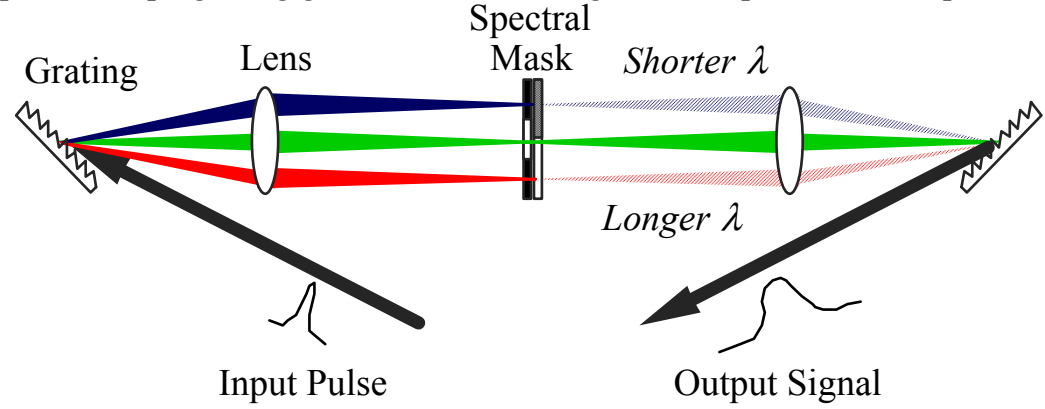

Figure 5: Longitudinal laser profile shaping by controlling phase and amplitude modulation using phase mask

\section{PERFORMANCE OF THE INJECTOR}

The LCLS photo-injector will be operating at lower RF gun phase as required by the longitudinal emittance compensation (fig. 6)[9,10]. One of the consequence of operating at lower RF gun phase is timing jitter will lead to intensity jitter due to the Schottky effect. The ATF operation experience shows that, sub pico-second peak to peak timing jitter is required. Based on the recent ATF experimental results, we think that LCLS injector should be operating at a charge of $0.5 \mathrm{nC}$ and peak current $100 \mathrm{~A}$.

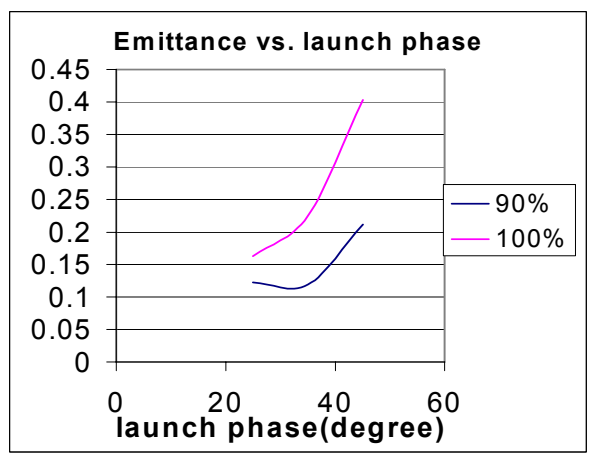

Figure 6: Emittance as function of RF gun phase for $1 \mathrm{nC}$.

\section{ACKNOWLEDGEMENT}

Authors would like to thank our SLAC colleague for useful discussions, particular with Dr J. Clendenin on LCLS injector requirements. The support from NSLS and BNL director office are greatly appreciated.

\section{REFERENCES}

[1] M. Cornacchihia et al, "Linac Coherent Light Source (LCLS) Design Study Report", SLAC-R-521/UC-414 (1998).

[2] S. Sakai et al, "Development of High-Duty Operation RF photoinjector", BNL-65003 (1997).

[3] S. Milton et al, "Exponential Gain and Saturation of a SASE FEL", to be published in Science.

[4] A. Tremaine et al, Saturation Measurements of VISA, to be published.

[5]X.J. Wang, "Single-Pass High-Gain FEL Beam Diagnostics", Proceeding Of Beam Instrumentation Workshop 2000, AIP Conf. Proc. 546, 164-185 (2000).

[6] A. Endo, SHI Pulrise laser system.

[7] X.Y. Chang, X.J. Wang and I. Ben-Zvi, this proceeding.

[8] K. TAKASAGOA et al, Square Laser Pulse Generation, presented at ICAF beam dynamics workshop, June 11-15, Stony Brook, USA.

[9] X.J Wang, X. Qiu and I. Ben-Zvi, Phys. Rev. E,54, R3121 - R3124 (1996).

[10] X.J. Wang and I. Ben-Zvi, Pro. Of PAC'97, 27932795(1997). 